\title{
INO1 transcriptional memory leads to DNA zip code- dependent interchromosomal clustering
}

\author{
Donna Garvey Brickner, Robert Coukos and Jason H. Brickner* \\ Department of Molecular Biosciences, Northwestern University, Evanston, IL USA 60201. \\ * Corresponding Author: Jason H. Brickner, E-mail: j-brickner@northwestern.edu
}

\begin{abstract}
Many genes localize at the nuclear periphery through physical interaction with the nuclear pore complex (NPC). We have found that the yeast INO1 gene is targeted to the NPC both upon activation and for several generations after repression, a phenomenon called epigenetic transcriptional memory. Targeting of INO1 to the NPC requires distinct cis-acting promoter DNA zip codes under activating conditions and under memory conditions. When at the nuclear periphery, active INO1 clusters with itself and with other genes that share the GRS I zip code. Here, we show that during memory, the two alleles of INO1 cluster in diploids and endogenous INO1 clusters with an ectopic INO1 in haploids. After repression, INO1 does not cluster with GRS I containing genes. Furthermore, clustering during memory requires Nup100 and two sets of DNA zip codes, those that target INO1 to the periphery when active and those that target it to the periphery after repression. Therefore, the interchromosomal clustering of INO1 that occurs during transcriptional memory is dependent upon, but mechanistically distinct from, the clustering of active INO1. Finally, while localization to the nuclear periphery is not regulated through the cell cycle during memory, clustering of INO1 during memory is regulated through the cell cycle.
\end{abstract}

\author{
doi: $10.15698 / \operatorname{mic} 2015.12 .242$ \\ Received originally: 01.09.2015; \\ in revised form: 24.10.2015, \\ Accepted 27.10.2015, \\ Published 13.11.2015.
}

Keywords: epigenetic inheritance, transcriptional memory, nuclear pore, DNA zip code, interchromosomal clustering.

\section{Abbreviations:}

GRS - gene recruitment sequence, LacO - Lac operator,

MRS - memory recruitment sequence, NPC - nuclear pore complex, Nups - nuclear pore proteins, TetO - Tet operator.

\section{INTRODUCTION}

Eukaryotic genomes are spatially organized. Chromosomes compact, form intrachromosomal loops and interact with each other and with subnuclear structures [1]. Such interactions lead to stereotypical arrangements of chromosomes with respect to each other and with respect to nuclear landmarks.

Individual genes often change their position when induced or repressed. A well-studied phenomenon that illustrates this point and that serves as an excellent model is the movement of yeast genes from the nucleoplasm to the nuclear periphery upon activation [2, 3]. Inducible genes such as INO1, GAL1-10, GAL2, TSA2, HSP104 and HXT1 move to the nuclear periphery and physically interact with the NPC upon activation [2-7]. Mutations in nuclear pore proteins (Nups) block targeting to the periphery $[6,8-10]$ and genome-wide ChIP experiments in yeast, flies, and mammalian cells indicates that hundreds to thousands of genes interact with NPCs or nuclear pore proteins [3, 1114]. Thus, interaction with the NPC leads to changes in gene positioning.
Interaction of yeast genes with the NPC and positioning to the nuclear periphery requires small cis-acting DNA elements in their promoters $[6,7,10]$. For example, two elements called GRS I and GRS II in the INO1 promoter are necessary for targeting to the NPC (Figure 1A; ref. [6]). These elements function as DNA zip codes: they are both necessary for INO1 targeting and, when inserted at an ectopic site in the genome, they are sufficient to induce repositioning to the nuclear periphery and interaction with the NPC. The GRS I element binds to the Put3 transcription factor, which is required for GRS I-mediated positioning [7]. Thus, genomes encode subnuclear positioning through transcription factor binding sites that function as DNA zip codes.

In addition to promoting interaction with the NPC, DNA zip codes like GRS I promote interchromosomal clustering of genes [7]. The two alleles of INO1 in diploid cells cluster together upon activation. In haploids, INO1 clusters with another GRS I-targeted gene, TSA2 and with GRS I inserted at an ectopic locus [7]. Mutations in GRS I, loss of Nups or loss of Put3 disrupt interchromosomal clustering. Therefore, DNA zip codes such as the GRS I, are necessary and 
A
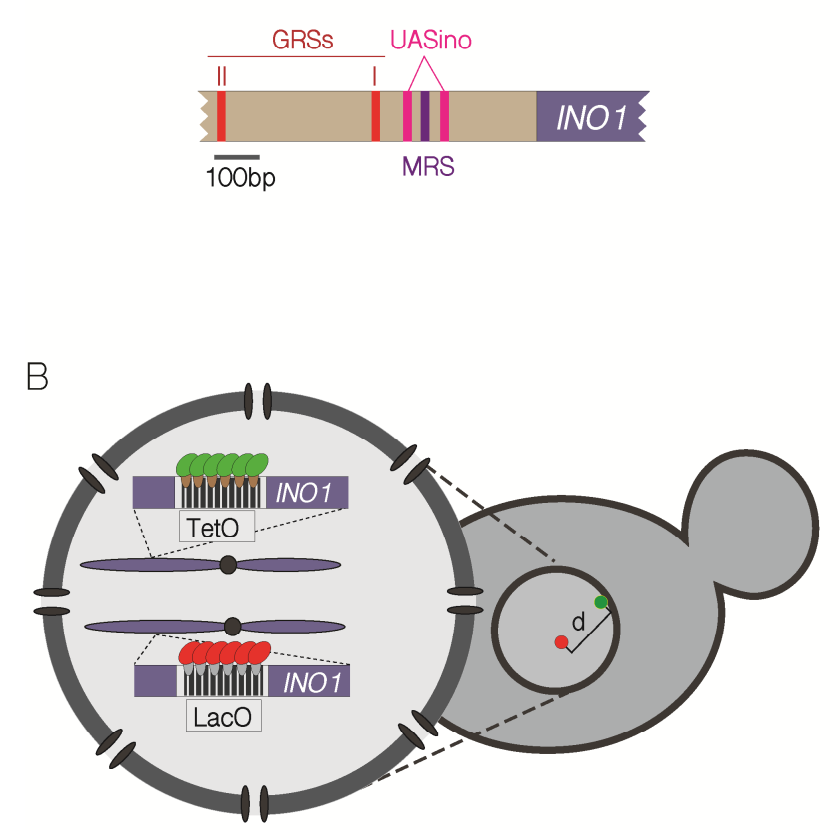
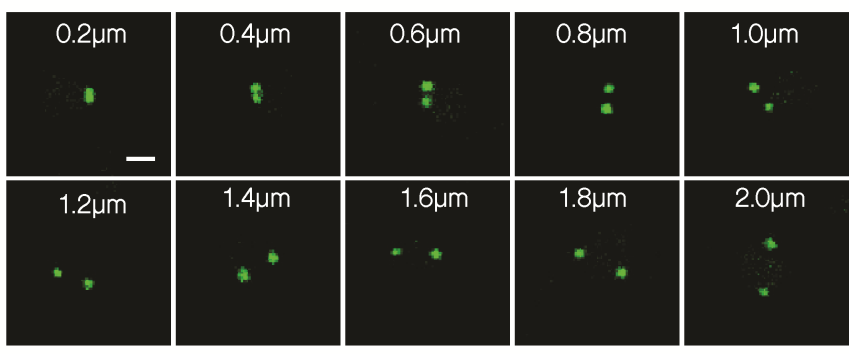

C

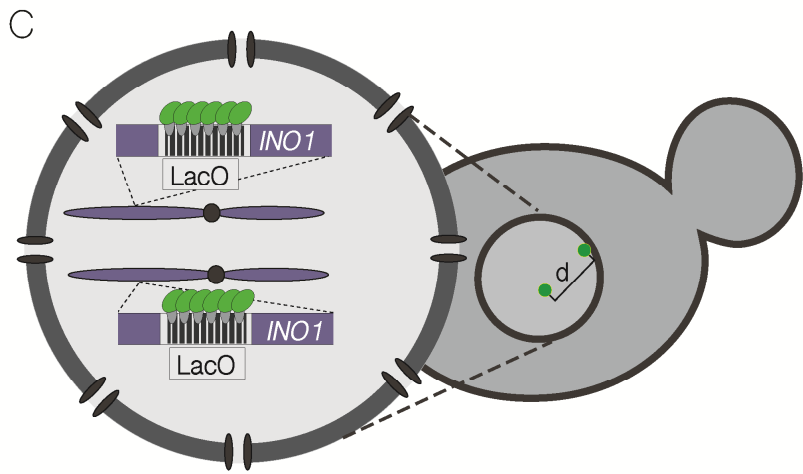

FIGURE 1: Experimental system. (A) Schematic of the INO1 promoter, with the relevant regulatory elements and DNA zip codes highlighted. GRS: Gene Recruitment Sequence [6]; MRS: Memory Recruitment Sequence [10]; UAS ${ }_{\mathrm{NNO}}$ : Upstream Activating Sequence regulated by inositol. (B and C) Experimental setups for studying interchromosomal clustering using two different repressor arrays (B) or two identical arrays (C). (D) Representative confocal micrographs of cells having two GFP-marked arrays. Scale bar $=1 \mu \mathrm{m}$.

sufficient to induce interchromosomal clustering of active loci through interaction with transcription factors and the NPC.

Upon repression, the INO1 gene remains associated with the NPC for several generations, a phenomenon called epigenetic transcriptional memory [8]. This interaction involves a different cis-acting DNA zip code (the Memory Recruitment Sequence, MRS; Figure 1A) and different nuclear pore proteins (e.g. Nup100; refs [10], [15]). Mutations in the GRS I and II elements do not affect targeting to the NPC during memory and mutations in the MRS do not affect targeting to the NPC during activation, suggesting that these two mechanisms are independent [8]. Here, we show that transcriptional memory also leads to interchromosomal clustering. Clustering during memory requires both previous clustering of active INO1 and the MRS zip code. However, unlike under activating conditions, INO1 does not cluster with the GRS I at an ectopic site under memory conditions. Therefore, INO1 clusters with different partners under activating and memory conditions, suggesting that interchromosomal clusters are remodeled upon repression. Finally, unlike targeting to the periphery [16], INO1 clustering is regulated through the cell cycle under both activating and memory conditions. These results show that interchromosomal clustering of INO1 during transcriptional memory is zip code-dependent but represents a molecular event that is distinct from targeting to the NPC.

\section{RESULTS}

\section{INO1 clustering during transcriptional memory}

To monitor clustering of INO1, we utilized several experimental systems: diploid strains in which one allele of INO1 is marked with an array of 128 Lac Operator (LacO) repeats and the other allele is marked with an array of 112 Tet Operator (TetO) repeats (Figure 1B), diploid strains in which both alleles of INO1 are marked with LacO (Figure 1C) or haploid strains in which INO1 is marked with TetO and other sites (i.e. URA3 or GAL1) are marked with LacO (similar to the system shown in Figure 1B). The distance between the two genes can be measured in each cell in a population (Figure 1D) and clustering can be assessed by comparing the distribution of distances between the two loci in the population or by measuring the fraction of cells in which the two loci are $\leq 0.55 \mu \mathrm{m}$ apart [7].

We previously showed that INO1 clusters with an ectopic copy of INO1 integrated near the URA3 locus in a haploid cell upon recruitment to the nuclear periphery [7]. To test if this clustering is maintained during memory, we compared the distances between INO1-TetO and URA3:INO1-LACO under long-term repressing conditions (overnight, +inositol) and under memory conditions (-inositol $\rightarrow$ +inositol, 3h). Under long-term repressing conditions, INO1 and URA3:INO1 do not obviously cluster together, showing a broad distribution with a mean distance of $0.85 \pm 0.38 \mu \mathrm{m}$ and $20 \% \leq 0.55 \mu \mathrm{m}$ (Figure $2 \mathrm{~A}$ ). This is similar to the distribution and clustering observed for two unrelated loci in haploid nuclei [7]. However, under 
A

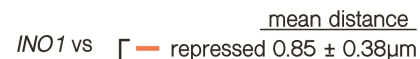
URA3:INO1 [ - memory $0.64 \pm 0.33 \mu \mathrm{m}$

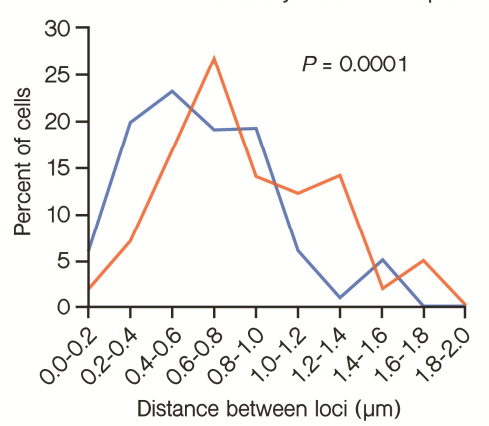

C
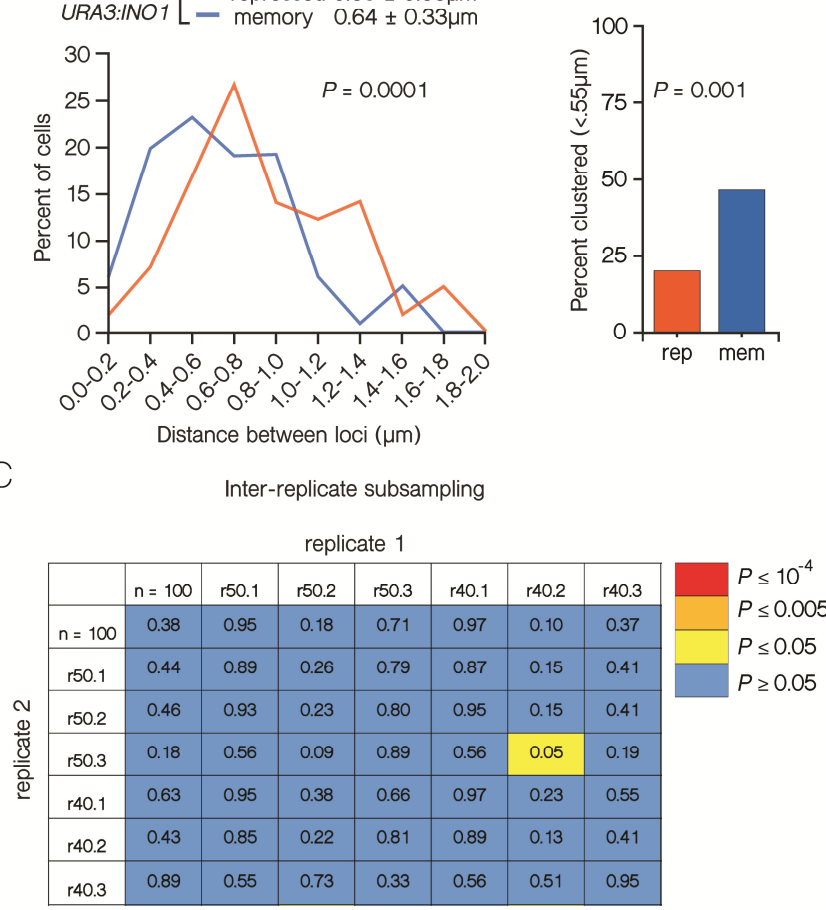

B

\begin{tabular}{|c|c|c|c|c|c|c|c|}
\hline & $n=100$ & $r 50.1$ & $r 50.2$ & $r 50.3$ & $r 40.1$ & $r 40.2$ & $r 40.3$ \\
\hline$n=100$ & & 0.59 & 0.76 & 0.57 & 0.75 & 0.46 & 0.85 \\
\hline$r 50.1$ & & & 0.48 & 0.94 & 0.88 & 0.27 & 0.56 \\
\hline$r 50.2$ & & & & 0.50 & 0.60 & 0.74 & 0.91 \\
\hline$r 50.3$ & & & & & 0.84 & 0.26 & 0.55 \\
\hline$r 40.1$ & & & & & & 0.37 & 0.65 \\
\hline$r 40.2$ & & & & & & & 0.68 \\
\hline$r 40.3$ & & & & & & & \\
\hline
\end{tabular}

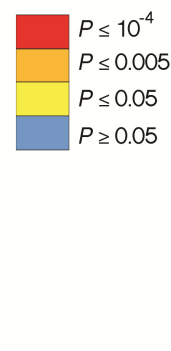

D

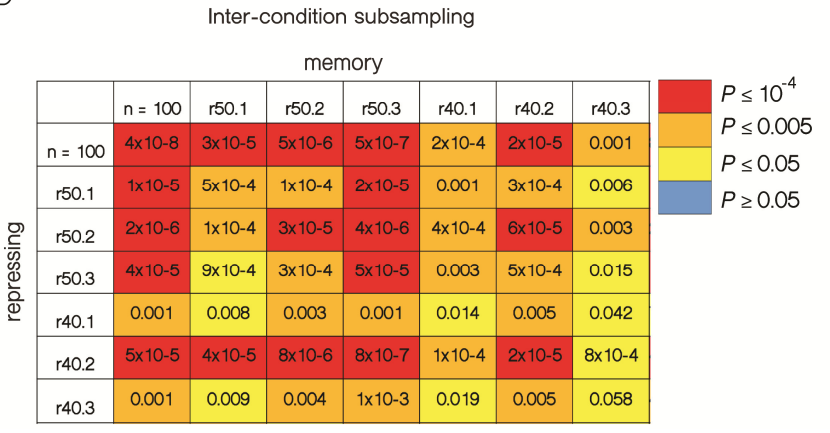

FIGURE 2: INO1 transcriptional memory leads to interchromosomal clustering. (A) Haploid cells having the endogenous INO1 gene marked with the TetO and URA3:INO1 marked with the LacO, expressing GFP-TetR and mRFP-Lacl [8] were grown under INO1 repressing (+ inositol) or memory (- inositol $\rightarrow+$ inositol, $3 \mathrm{~h}$ ) conditions, fixed and processed for immunofluorescence against GFP and mRFP. Left: The distribution of distances between the two loci in 100 cells, binned into $0.2 \mu \mathrm{m}$ bins. $P$ values were calculated using a Wilcoxon Rank Sum Test. Right: the fraction of cells in which the two loci were $\leq 0.55 \mu \mathrm{m}$. $P$ values were calculated using a Fisher Exact Test. Note: the distribution of the repressed condition has been previously published [7] and is shown only for comparison to the distribution under the experimental (memory) condition. (B-D) Subsampling analysis. Full datasets $(n=100)$ or randomly generated subsamples of 50 or 40 measurements (r50 or r40, respectively) were compared pairwise using a Wilcoxon Rank Sum test. The numbers in each cell are the $P$ values, color-coded as described in the legend. (B) A biological replicate compared with itself. (C) Two biological replicates compared with each other. (D) Distributions from repressing and memory conditions compared with each other.

memory conditions, there is a significant shift in the distribution to shorter distances (mean $=0.64 \pm 0.33 \mu \mathrm{m} ; P=$ 0.0001 , Wilcoxon Rank Sum test) and an increase in the fraction of cells in which the two loci are $\leq 0.55 \mu \mathrm{m}(47 \% ; P$ $=0.001$, Fisher Exact test; Figure 2A). Thus, endogenous INO1 remains clustered with an ectopic copy of INO1 under memory conditions. For the experiments that follow (Figure $3,4 \mathrm{~A}$ ), this distribution served as a control for comparison.

To assess the variance and sample size, we subjected the data to additional analysis. First, we collected three random subsamples (of 50 or 40 cells each; labeled r50 and $r 40$ in Figure 2) from the data that were used to generate the distribution of distances under memory conditions ( $n=$ 100 cells) and compared them to the total dataset and to each other using a Wilcoxon Rank Sum test. This analysis showed that there is no significant difference between the total data and subsets of the data of $\geq 40$ cells (Figure $2 B$ ).

To assess the variance between biological replicates, we performed the analysis above using total datasets $(n \geq$ 100 cells) or random subsamples from two independent biological replicates (Figure 2C). Of the 49 comparisons, only one (a subset of 40 compared with a subset of 50 ) was significantly different $(P=0.05$; Figure $2 C$ ). In every comparison in which one of the two datasets was complete ( $\mathrm{n}$ $\geq 100$ ) or in which both datasets contained $\geq 50$ cells, no significant differences were observed (Figure $2 \mathrm{C}$ ). This suggests that data from $\geq 100$ cells are oversampled and sufficient to avoid Type I errors (i.e. incorrect rejection of the null hypothesis that two datasets are the same).

Finally, to assess statistical power and sensitivity, we used random subsampling to compare two datasets from different conditions (repressing vs. memory conditions; Figure 3D). This analysis revealed that all sets of measurements containing $\geq 40$ measurements were sufficient to reveal statistically significant difference and that the significance of the difference was greater when more cells were analyzed (Figure 3D). Therefore, comparing two datasets of 40 cells is sufficient to avoid Type II errors (failing to reject the null hypothesis that the two datasets are same), so we have measured $\sim 100$ cells per experiment in the work described here. 


\section{INO1 clustering during transcriptional memory is specific and MRS-dependent}

To confirm that clustering under memory conditions is specific, we examined the positioning of INO1 (marked with TetO) with respect to GAL1 (marked with LacO) after simultaneously repressing both genes (Figure $3 \mathrm{~A}$ ). Both INO1 and GAL1 exhibit transcriptional memory, localizing at the nuclear periphery for several generations after repression [8]. However, unlike INO1 and URA3:INO1, INO1 and GAL1 did not cluster under memory conditions (Figure $3 \mathrm{~A} ; 15 \% \leq 0.55 \mu \mathrm{m})$. Therefore, the clustering of INO1 with itself under memory conditions is specific.

The MRS zip code is specifically required for localization of INO1 at the nuclear periphery during memory and has no role in targeting of active INO1 to the nuclear periphery [10]. Mutation of the MRS disrupts peripheral localization under memory conditions. Therefore, we asked if the MRS is necessary for clustering by measuring the distances between INO1 and URA3:INO1 having a mutation in the MRS element ( $m r s$ INO1) under repressing and memory conditions (Figure 3B). Mutation of the MRS disrupted INO1 clustering during memory $(13 \% \leq 0.55 \mu \mathrm{m})$, indicating that the clustering of INO1 with URA3:INO1 after repression requires the MRS zip code.

The MRS is both necessary and sufficient to promote targeting to the nuclear periphery under memory conditions [10]. To test if the MRS is sufficient to induce cluster-
A

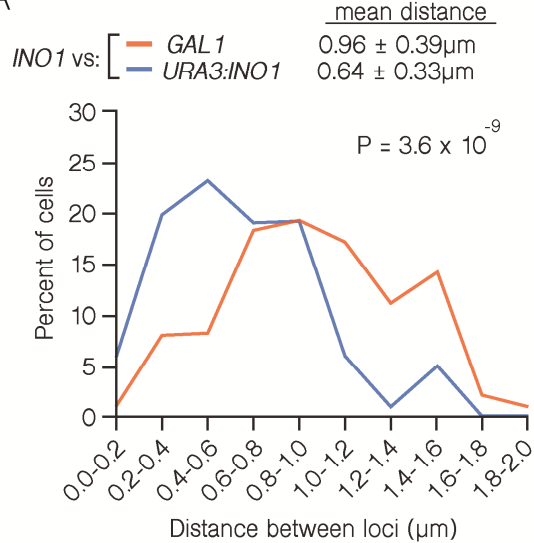

C

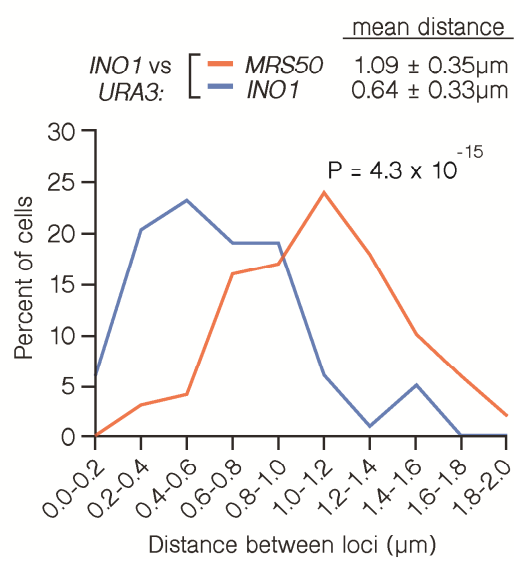

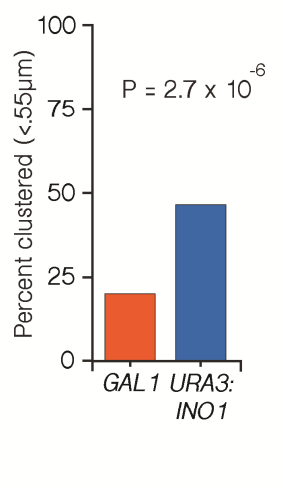

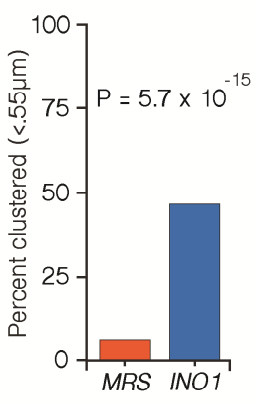

ing with INO1 during memory, we compared the positions of INO1 and URA3:MRS5O (a 50 bp element including the MRS; ref. [10]) during memory. The MRS50 was not sufficient to induce clustering with the endogenous INO1 gene (Figure 3C; $6 \% \leq 0.55 \mu \mathrm{m}$ ). This suggests that additional information besides the MRS is provided by the INO1 gene to promote clustering during transcriptional memory.

\section{A hierarchy of DNA zip codes controls INO1 clustering during transcriptional memory}

To explore what other signals might be important for clustering of INO1 during transcriptional memory, we tested other DNA zip codes. Targeting of active INO1 to the nuclear periphery requires the GRS I and GRS II zip codes and mutation of both elements blocks targeting of active INO1 to the nuclear periphery and disrupts interchromosomal clustering (Figure $1 \mathrm{~A}$ and ref. [7]). However, these mutations do not affect targeting of INO1 to the nuclear periphery during memory, indicating that MRS-mediated targeting to the nuclear periphery is independent of GRSmediated targeting [10]. Because the MRS was necessary but not sufficient to induce clustering during memory, we hypothesized that the interchromosomal interactions of active genes might be required for the persistent clustering after repression.

To test this proposal, we examined the interaction between wild type INO1 and URA3:INO1 having mutations in
B

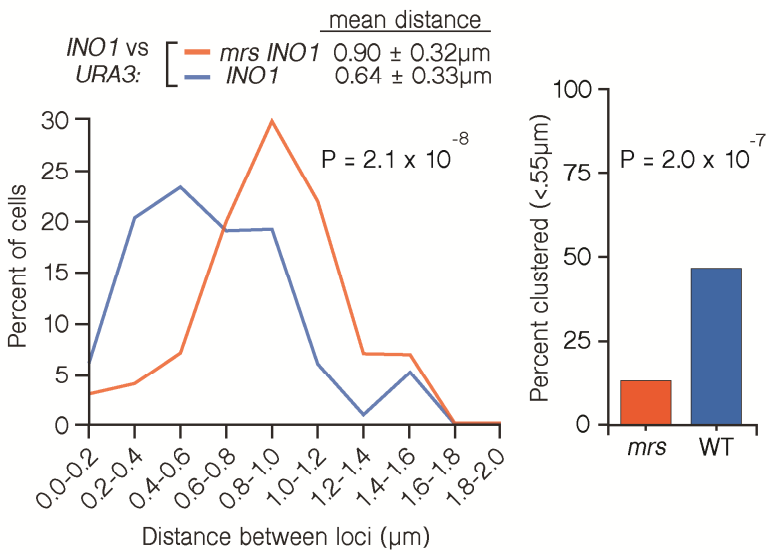

FIGURE 3: INO1 interchromosomal clustering during memory is specific and MRS-dependent. (A-C) Haploid cells having the endogenous INO1 gene marked with the TetO and either URA3 (AC) or GAL1 (A) marked with the LacO, expressing GFP-TetR and mRFP-Lacl [8] were grown under INO1 memory (activating $\rightarrow$ repressing, $3 \mathrm{~h}$ ) conditions, fixed and processed for immunofluorescence against GFP and mRFP. Left: The distribution of distances between the two loci in $\sim 100$ cells, binned into $0.2 \mu \mathrm{m}$ bins. $P$ values were calculated using a Wilcoxon Rank Sum Test. Right: the fraction of cells in which the two loci were $\leq 0.55 \mu \mathrm{m}$. $P$ values were calculated using a Fisher Exact Test. Note that the data used to generate the distribution for the control of INO1-TetO vs. URA3:INO1-LaCO is the same in all three panels. The combinations tested were INO1-TetO vs. URA3:INO1-LacO (A-C), or INO1TetO vs. GAL1-LaCO (A), INO1-TetO vs. URA3:mrsINO1-LacO (B) and INO1-TetO vs. URA3:MRS5O-LaCO (C). 
the GRS I and GRS II (grs1,2 INO1). In this strain, clustering is lost under both activating [8] and memory conditions (Figure $4 \mathrm{~A} ; 17 \% \leq 0.55 \mu \mathrm{m}$ ). Therefore, even though targeting to the nuclear periphery during transcriptional memory is GRS I- and GRS II-independent, clustering of INO1 during memory is GRS I- and GRS II-dependent. This suggests that targeting to the nuclear periphery and interchromosomal clustering during memory must represent distinct molecular events.

Although both GRS I and GRS II are capable of mediating targeting to the nuclear periphery, GRS I directs interchromosomal clustering of INO1 with itself and GRS II does not [8]. This suggests that targeting to the periphery is necessary, but not sufficient, to promote interchromosomal clustering. Because the loss of GRS I and GRS II disrupted clustering of INO1 during transcriptional memory, we next asked if this is due to loss of peripheral targeting under activating conditions or due to loss of interchromosomal clustering. Mutants that lack the GRS I - binding pro- tein Put3 still target INO1 to the nuclear periphery normally (due to GRS II function), but fail to cluster [8]. We tested the effect of loss of Put3 on INO1 clustering during memory (Figure 4B). As expected, clustering of active INO1 with URA3:INO1 was disrupted in put3 $\triangle$ mutants (Figure $4 \mathrm{~B})$. Consistent with the idea that clustering of active INO1 being required for clustering of recently repressed INO1, loss of Put3 also disrupted clustering of INO1 with URA3:INO1 during memory (Figure 4B).

This result led us to ask if the GRS I alone, which is sufficient to induce clustering with active INO1, or GRS II, which is not [8], could also induce clustering with INO1 during memory conditions. Consistent with our previously published work, active INO1 clusters strongly with URA3:GRS I (ref. [7]; Figure 4C; $58 \% \leq 0.55 \mu \mathrm{m}$ ) but not with URA3:GRS II (Figure 4D; 13\% $\leq 0.55 \mu \mathrm{m}$ ). INO1 did not cluster with either URA3:GRS I (Figure 4C; $11 \% \leq 0.55 \mu \mathrm{m}$ ) or URA3:GRS II (Figure 4D; 15\% $\leq 0.55 \mu \mathrm{m}$ ) under memory conditions. Therefore, neither the GRS I nor the GRS II are
A

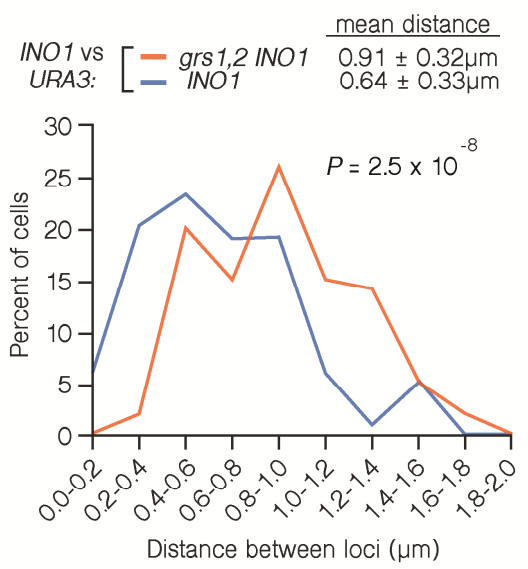

C

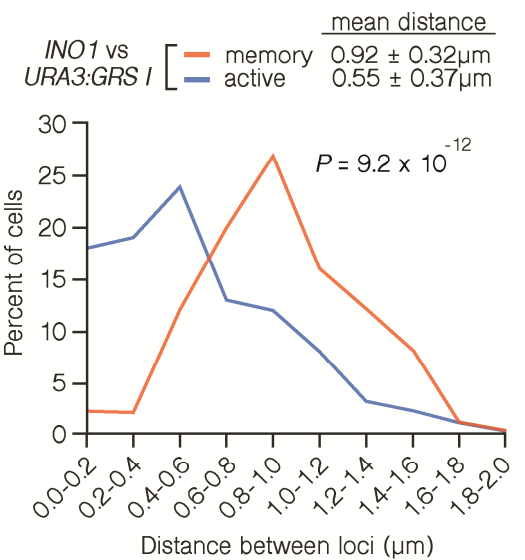

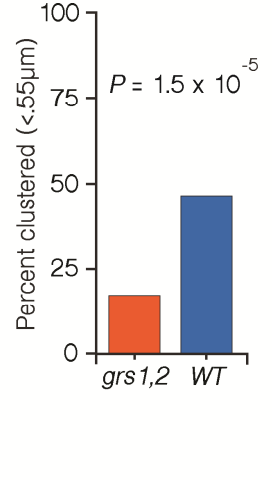

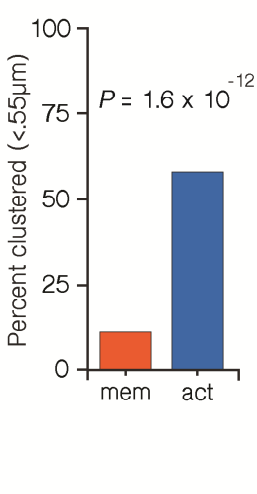

B
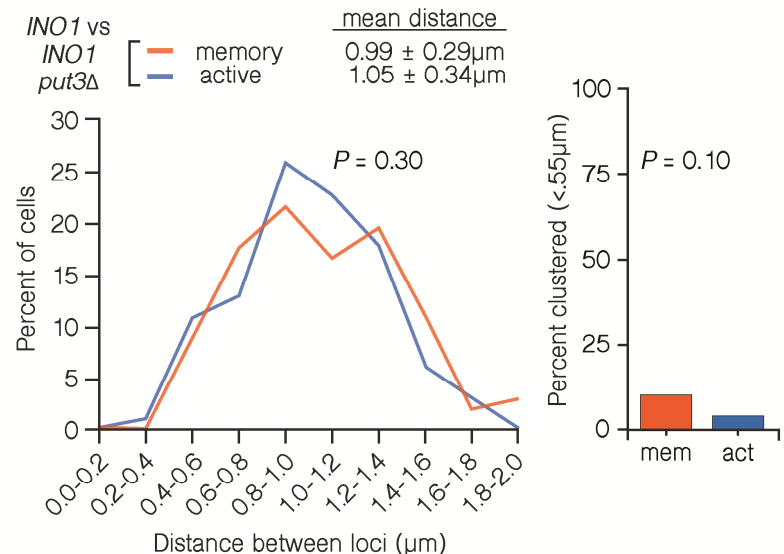

Distance between loci $(\mu \mathrm{m})$

D
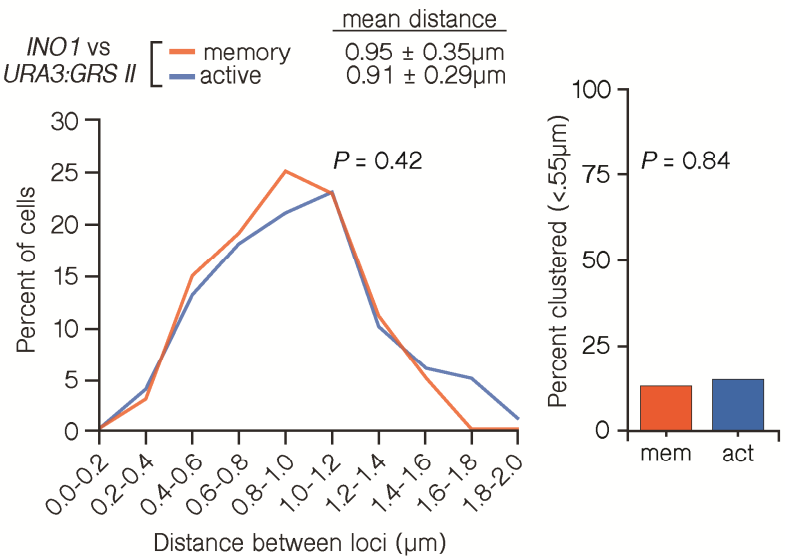

FIGURE 4: INO1 interchromosomal clustering during transcriptional memory requires clustering of active INO1. (A and B) Haploid cells having the LacO array integrated at URA3 and the TetO array integrated at INO1, and expressing GFP-TetR and mRFP-Lacl were fixed and processed for immunofluorescence against GFP and mRFP. Left: The distribution of distances between the two loci in 100 cells, binned into $0.2 \mu \mathrm{m}$ bins. $P$ values were calculated using a Wilcoxon Rank Sum Test. Right: the fraction of cells in which the two loci were $\leq 0.55 \mu \mathrm{m}$. $P$ values were calculated using a Fisher Exact Test. (A) INO1-TetO vs. URA3:INO1-LacO or URA3:grs1,2 INO1-LacO grown under memory conditions. (B) INO1-TetO vs. URA3:GRS I-LaCO under either activating or memory conditions. (C) INO1-TetO vs URA3:GRS II-LacO under activating or memory conditions. (D) INO1-TetO vs. URA3:INO1-LacO put3A cells under activating or memory conditions. 
sufficient to induce clustering with INO1 during memory and the clustering of INO1 with URA3:GRS I is not stably maintained after INO1 repression.

\section{Interchromosomal clustering of INO1 during transcrip-} tional memory requires Nup100

The interaction of INO1 with the NPC is different under activating and memory conditions. The interaction of the NPC with these two states requires different cis-acting DNA zip codes and different Nups [10]. One such protein is Nup100; mutants lacking Nup100 target INO1 to the periphery under activating conditions but not under memory conditions [10].

To explore the role of the nuclear pore in clustering of INO1 during transcriptional memory, we measured the distances between INO1 alleles (marked with LacO and TetO; Figure 1B) in wild type or nup $100 \Delta /$ nup $100 \Delta$ diploid cells grown under activating, repressing and memory conditions. Under both activating or memory conditions in the wild type (NUP100/NUP100) diploid cells, INO1 clustered to very similar extent (Figures 5A and 5B). Nup100 is dispensable for clustering of active INO1 alleles; in diploid cells lacking Nup100, the two active alleles of INO1 clustered together (Figure 5C; mean distance $=0.74 \pm 0.43 \mu \mathrm{m}$; $51 \% \leq 0.55 \mu \mathrm{m})$. However, Nup100 is specifically required for INO1 clustering during memory; under memory conditions, INO1 clustering was lost in nup $100 \Delta /$ nup $100 \Delta$ diploid cells (Figure 5D; mean distance $=1.11 \pm 0.52 \mu \mathrm{m} ; 18 \% \leq$ $0.55 \mu \mathrm{m})$.

\section{Cell cycle regulation of interchromosomal clustering of} INO1 during memory

Positioning of active INO1, GAL1 and HSP104 at the nuclear periphery is regulated through the cell cycle. Immediately after the initiation of DNA replication, all three of these genes reposition to the nucleoplasm for $\sim 30$ minutes before returning to the periphery during mitosis $[16,17]$. The clustering of active INO1 with itself is maintained during Sphase in the nucleoplasm [7]. Thus, the cell cycle regulation of peripheral localization is uncoupled from interchromosomal clustering.

In contrast to the active genes, during transcriptional memory, INO1 and GAL1 remain at the nuclear periphery throughout the cell cycle [16]. Thus, it was unclear if clus-
A
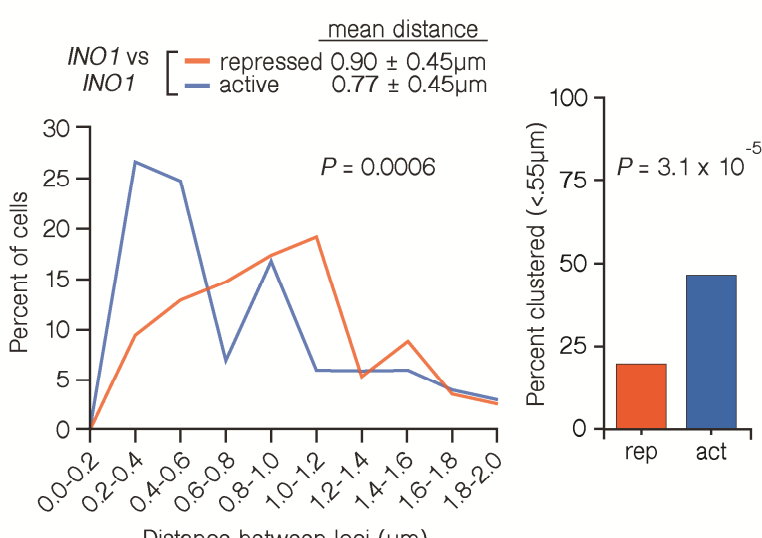

C

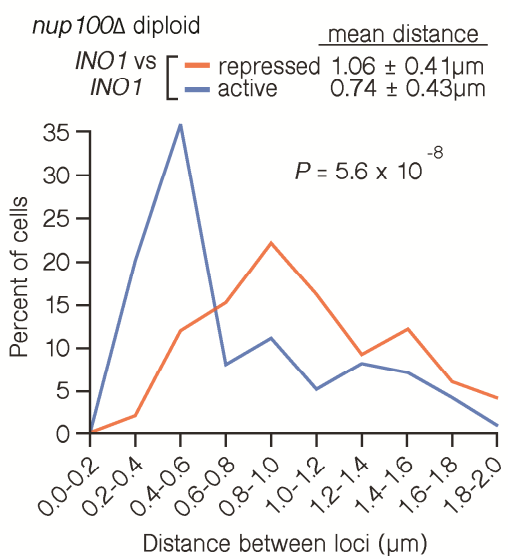

B
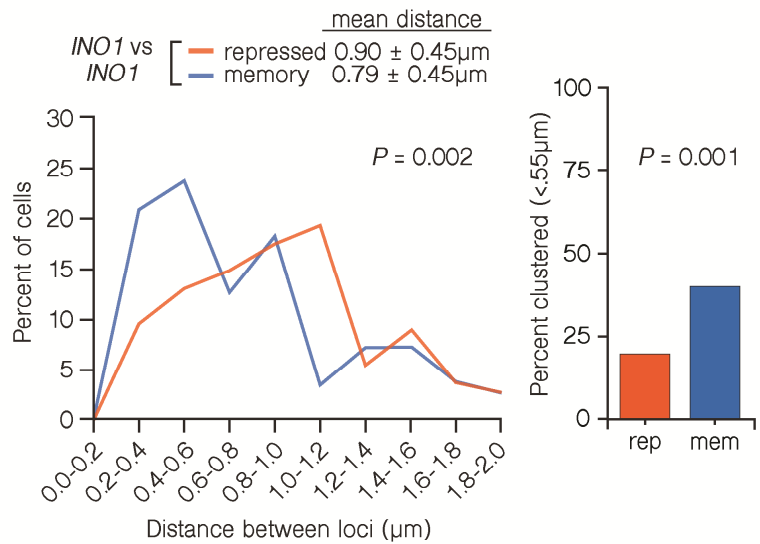

Distance between loci $(\mu \mathrm{m})$

D

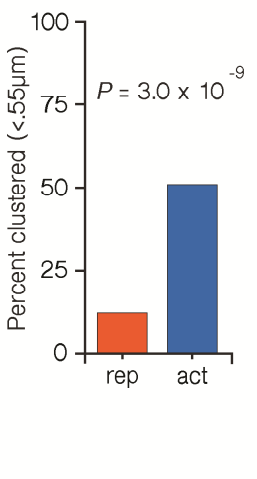

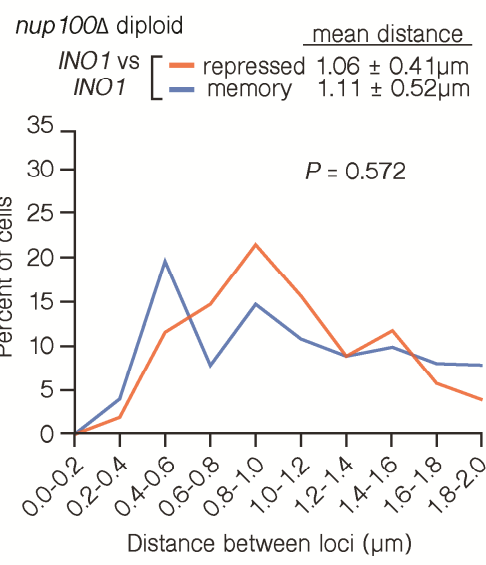

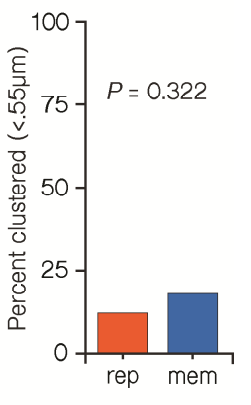

FIGURE 5: Nup100 is specifically required for INO1 clustering during memory. Diploid cells having both alleles of INO1 marked with the LacO array and expressing GFP-Lacl were grown under repressing, activating or memory conditions. Left: The distribution of distances between the two loci in $~ 100$ cells, binned into $0.2 \mu \mathrm{m}$ bins. $P$ values were calculated using a Wilcoxon Rank Sum Test. Right: the fraction of cells in which the two loci were $\leq 0.55 \mu \mathrm{m}$. P values were calculated using a Fisher Exact Test. (A and B) NUP100/NUP100 diploids. (C and D)

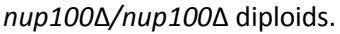


tering would also be maintained through the cell cycle. We tested this idea by comparing the \% of unbudded (G1), small budded (S) and large budded (G2) cells in an asynchronous population in which the two alleles were $\leq 0.55$ $\mu \mathrm{m}$ (Figure 6A). We utilized a diploid strain in which both copies of INO1 were marked with LacO arrays bound to GFP-Lacl (Figure 1C and 1D). This experimental setup allows rapid 3D mapping of the two alleles in live cells. However, because both spots are the same color, it is not possible to score cells in which the spots are unresolvable (i.e. only a single spot is observed), which includes some cells in which the two loci are $\leq 0.2 \mu \mathrm{m}$ apart. Despite this limitation, we could observe clustering of INO1 alleles under both activating (Figure $6 \mathrm{~A}$ ) and memory conditions (Figure $6 \mathrm{~B})$ using this system.

Distances between the two alleles of INO1 were measured in unbudded $\mathrm{G} 1$, small budded S-phase and large budded G2 cells under memory conditions (Figure 6C). In unbudded cells and in small budded, we observed high level clustering $(57 \% \leq 0.55 \mu \mathrm{m}$; Figure $6 \mathrm{D})$. However, clus- tering of INO1 was lost in large budded cells $(9 \% \leq 0.55 \mu \mathrm{m}$; Figure $6 \mathrm{D}$ ). In these cells, which have not yet undergone nuclear division, each allele of recently repressed INO1 remains at the nuclear periphery [16], paired with its sister chromatid. However, the two alleles of INO1 do not cluster together, suggesting that clustering during memory is uncoupled from gene positioning to the periphery.

\section{DISCUSSION}

Interchromosomal clustering is a common phenomenon in eukaryotic cells. In budding yeast, as in many organisms, the 32 telomeres cluster into several foci at the nuclear periphery [18]. Likewise, the $\sim 250$ tRNA genes, scattered throughout the genome, form two clusters, one in the nucleolus and the other near the spindle pole body [19]. In flies, Polycomb repressed loci cluster together into Polycomb bodies $[20,21]$. And in mammalian cells, coregulated genes frequently co-localize within the nucleus [22-24]. Thus, cells frequently utilize interchromosomal clustering as a mechanism of spatial, and perhaps tran-
A

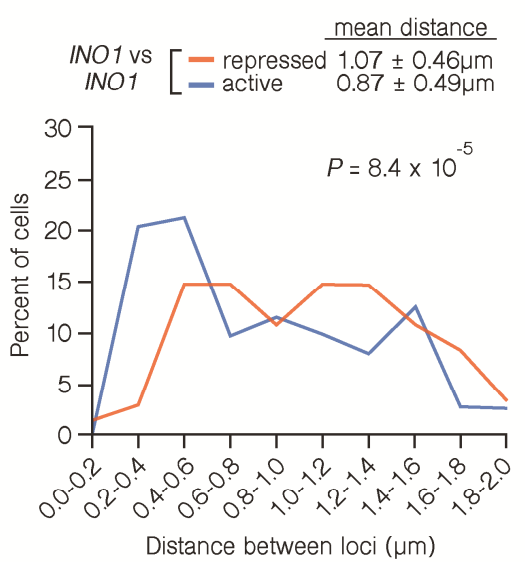

C

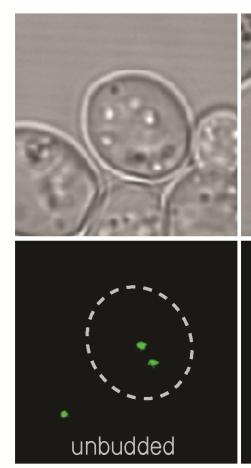

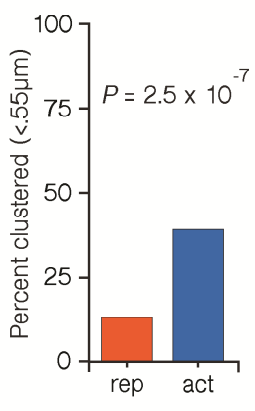

B
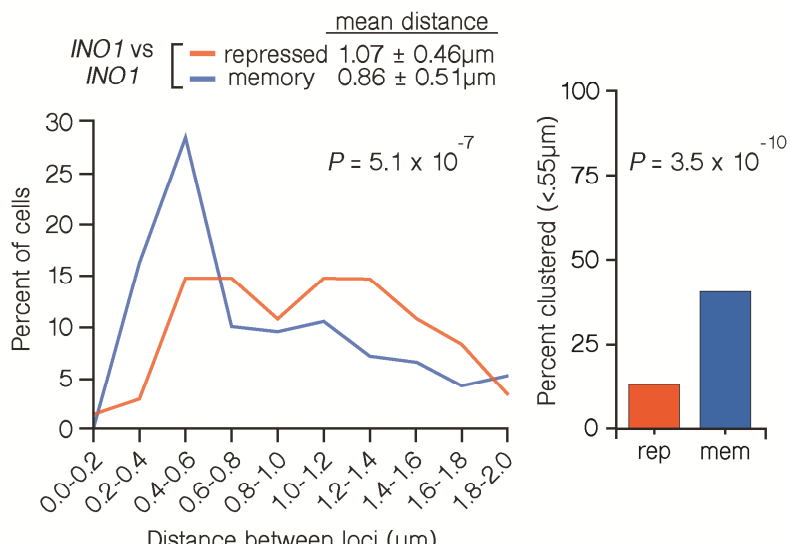

FIGURE 6: Interchromosomal clustering of recently repressed INO1 is regulated through the cell cycle. (A and B) An asynchronous population of cells having INO1:TetO and INO1:LacO, expressing GFP-TetR, GFP-Lacl and Pho88-mCherry (ER/nuclear envelope membrane protein) were grown under repressing, activating or memory conditions. Left: The distribution of distances between the two loci in 100 cells, binned into $0.2 \mu \mathrm{m}$ bins. $P$ values were calculated using a Wilcoxon Rank Sum Test. Right: the fraction of cells in which the two loci were $\leq 0.55 \mu \mathrm{m}$. $P$ values were calculated using a Fisher Exact Test. (C) Bright field (top) and green fluorescence (bottom) channels of typical cells used to measure distances in cells with different bud morphologies. The outline of the cell above is overlaid on the green channel (hatched line). (D) Cells were scored for both their bud morphology and the distance between the two loci and the fraction of each class of cells that was $\leq 0.55$ $\mu \mathrm{m}$ was determined. $P$ values were calculated using the Fisher Exact Test. 
scriptional, control.

Our previous work has established another mechanism by which interchromosomal clustering is facilitated. Interaction of active yeast genes with the NPC leads to both repositioning to the nuclear periphery and clustering with other loci that share the same zip code [7]. DNA zip codes are both necessary and sufficient to induce this type of interchromosomal clustering. It is unclear if clustering reflects targeting of genes to the same portion of the nuclear envelope or homotypic interactions between genes after they are targeted to an imprecise location on the envelope. Nups are essential for clustering, suggesting that targeting to the NPC is a prerequisite for clustering. However, once formed, the clusters can persist in the nucleoplasm [7]. This argues either that clustering is maintained by Nups that dissociate from the NPC, as has been shown to occur in many cell types, or that the NPC serves as a site of assembly for clusters but is not required after they are assembled.

Here we have explored a new model for interchromosomal clustering between genes that are poised for future activation. Of the genes that interact with the NPC when they are active, a small subset shows transcriptional memory, maintaining the interaction after repression (Figure 7A; ref. [8]). The Nups and the DNA zip codes required for memory are distinct from those required for peripheral localization and clustering of active genes (Figure 7A; ref. [10]). Here, we find that the INO1 gene remains clustered with itself after repression. However, the molecular mechanism of clustering during memory is very different from the mechanism of clustering of active INO1.

Active INO1 clusters both with itself and with other GRS I-containing genes [7]. Although we cannot rule out that INO1 clusters with other loci during memory, this mechanism is more selective than GRS I-mediated clustering (Figure 7B). The MRS zip code is necessary, but not sufficient (when inserted at an ectopic site), to induce clustering with INO1 during memory. Additional information besides the MRS is required for clustering.

In addition to the MRS, the GRS zip codes are required for INO1 clustering during memory, suggesting that clustering during memory requires previous clustering during the activating condition (Figure 7B). In other words, clustering during transcriptional memory reflects the previous clustering of active INO1. This is surprising because the mechanisms of targeting to the nuclear periphery when INO1 is active or during memory are completely independent [10]. Therefore, although interchromosomal clustering requires targeting to the nuclear periphery and is lost in cells in which either the zip code (MRS) or Nups (i.e. Nup100) are mutated, it has additional requirements and represents a different molecular mechanism.

Consistent with this idea, the regulation of interchromosomal clustering through the cell cycle does not perfectly mirror peripheral localization. Whereas targeting of active INO1 and other genes is lost briefly during S-phase, peripheral localization of INO1 during transcriptional memory is not regulated by the cell cycle [16]. In contrast, the interchromosomal clustering of INO1 alleles during
A

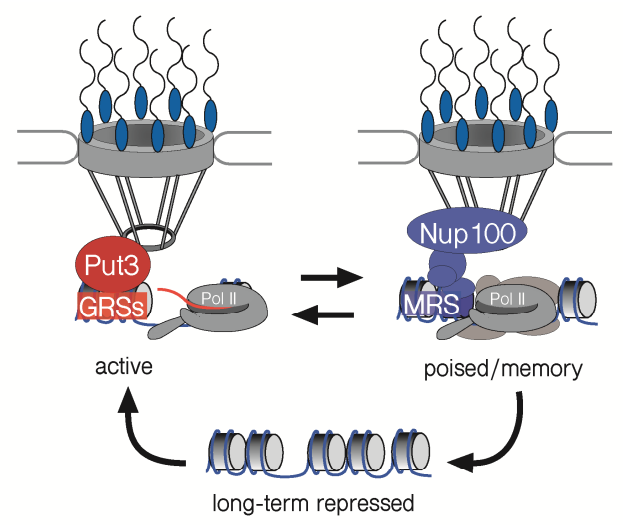

B

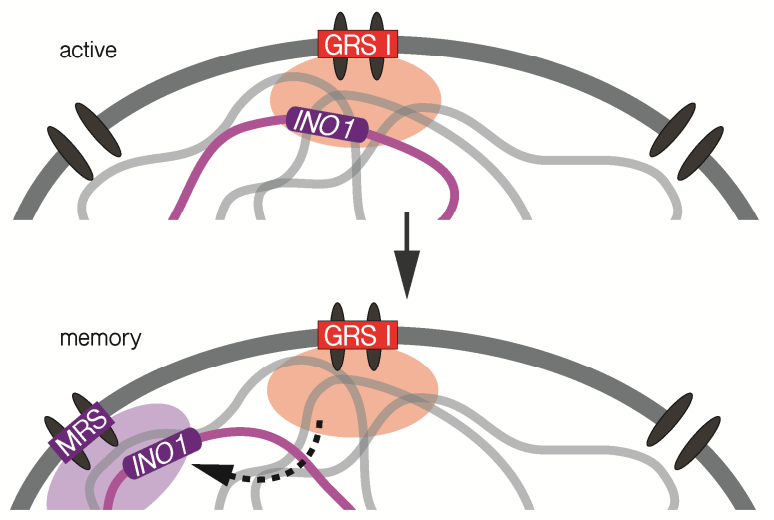

FIGURE 7: Model for zip code-dependent INO1 targeting to the NPC and interchromosomal clustering. (A) The long-term repressed gene is positioned in the nucleoplasm and both the active and recently repressed memory state of the gene are positioned at the nuclear periphery through interaction with the NPC. The GRS elements control targeting to the NPC under activating conditions. The Put3 transcription factor binds the GRS I zip code and is required for GRS I-mediated peripheral targeting and interchromosomal clustering [7]. The MRS element controls targeting to the NPC under memory conditions and requires Nup100 [10]. (B) The INO1 gene clusters with other GRS I-containing loci under activating conditions (top) and this is a prerequisite for clustering with itself (and potentially other loci) in an MRS-dependent cluster for several generations after repression, during transcriptional memory.

memory is lost during G2. This cell cycle regulation may reflect the coordination of interchromosomal clustering with chromosome condensation and chromosome segregation.

Most of the interchromosomal clustering events described previously are associated with either silenced sites like subtelomeric regions and Polycomb repressed sites or expressed sites like tRNA genes or induced genes. Here we show that a third class of genes, those that are repressed but poised for future induction, can also cluster in the nucleus. This interaction is specific to a moment in the life history of the INO1 gene: INO1 clusters with itself for 3-4 generations after repression, after which this is lost. Thus, INO1 epigenetic transcriptional memory, which leads to heritable changes in chromatin structure and binding of 
poised RNA Polymerase II to the promoter, also leads to heritable interchromosomal clustering.

\section{MATERIALS AND METHODS}

\section{Chemicals, yeast strains and growth conditions}

Chemicals were from Sigma Aldrich, molecular biology enzymes were from New England Biolabs and yeast growth media components were from Sunrise Science Products. For experiments involving scoring peripheral localization in live cells, PHO88 was C-terminally tagged with mCherry using PCR-based integration [25]. Briefly, the PHO88 termination codon was replaced by homologous recombination with a PCR product encoding a C-terminal translational fusion to mCherry, along with the His5+ gene from S. pombe. PCR primers were designed to incorporate 45 base pairs of sequences upstream and downstream of the termination codon. All yeast strains used in this study are described in Table 1 . Media, transformations and growth conditions are described in $[26,27]$.

\section{Microscopy}

For all experiments, cells were maintained at $\mathrm{OD}_{600}<0.5$. For inositol starvation experiments, strains were grown overnight in SDC-inositol in the presence or absence of $100 \mu \mathrm{M}$ myoinositol. To induce memory, inositol was added to $100 \mu \mathrm{M}$ and the cells were grown for an additional $3 \mathrm{~h}$. Immunofluorescence microscopy was carried out as described [7, 27]. For live cell experiments, cells were concentrated by brief centrifugation and imaged immediately. The images were captured as $0.34 \mu \mathrm{m}$ thick z-stacks with a Leica SP5 II Line Scanning Confo- cal Microscope with $100 \times 1.44$ NA (oil immersion) objective using an Argon $488 \mathrm{~nm}$ and Diode Pumped Solid State $561 \mathrm{~nm}$ lasers in the Northwestern Biological Imaging Facility as described [28]. Distances between the centers of each dot for cells in which the dots were in the same $\mathrm{z}$ slice were measured using LAS LiTE software.

\section{ACKNOWLEDGMENTS}

This work was supported by NIH grant GM080484 (JHB). JHB is the Sorretta and Henry Shapiro Research Professor in Molecular Biology. RC was supported by Undergraduate Research Grants from the Program in Biological Sciences and Northwestern University as well as a Krieghbaum Award. The authors thank members of the Brickner lab for helpful discussions and feedback on the manuscript.

\section{CONFLICT OF INTEREST}

The authors declare no conflict of interest.

\section{COPYRIGHT}

(C) 2015 Brickner et al. This is an open-access article released under the terms of the Creative Commons Attribution (CC BY) license, which allows the unrestricted use, distribution, and reproduction in any medium, provided the original author and source are acknowledged.

TABLE 1. Yeast strains used in this study.

\begin{tabular}{|c|c|c|}
\hline Strain & Genotype & Figures \\
\hline DBY326 & $\begin{array}{l}\text { MATa ade2-1 can1-100 his3-11,15 leu2-3,112 trp1-1 ura3-1 INO1:TetO-Nat LEU2:TetR-GFP } \\
\text { TRP1:Lacl-RFP GAL1:p6LacO128 }\end{array}$ & Figure $3 A$ \\
\hline DBY336 & $\begin{array}{l}\text { MATa ade2-1 can1-100 his3-11,15 leu2-3,112 trp1-1 ura3-1 INO1:TetO-Nat LEU2:TetR-GFP } \\
\text { TRP1:Lacl-RFP URA3:p6Lac0128-INO1 }\end{array}$ & Figs $2 A, 3 A-C, 4 A$ \\
\hline DBY339 & $\begin{array}{l}\text { MATa ade2-1 can1-100 his3-11,15 leu2-3,112 trp1-1 ura3-1 INO1:TetO-Nat LEU2:TetR-GFP } \\
\text { TRP1:Lacl-RFP URA3:p6LacOGRS } 241-270\end{array}$ & Figure 4C \\
\hline DBY348 & $\begin{array}{l}\text { MATa/MAT } \alpha \text { ade2-1/ade2-1 can1-100/can1-100 his3-11,15/his3-11,15 leu2-3,112/ } \\
\text { LEU2:TetR-GFP trp1-1/TRP1:Lacl-RFP ura3-1/URA3:p6LacO128-INO1 INO1/INO1:TetO-Nat }\end{array}$ & Figure 3C \\
\hline DBY354 & $\begin{array}{l}\text { MATa ade2-1 can1-100 his3-11,15 leu2-3,112 trp1-1 ura3-1 HIS3:Lacl-GFP Sec63-myc:TRP1 } \\
\text { LEU2:Lacl-GFP URA3:p6Lac0128 grs1,2mtIN01:p6Lac0128 }\end{array}$ & Figure $4 \mathrm{~A}$ \\
\hline DBY355 & $\begin{array}{l}\text { MATa ade2-1 can1-100 his3-11,15 leu2-3,112 trp1-1 ura3-1 INO1:TetO-Nat LEU2:TetR-GFP } \\
\text { TRP1:Lacl-RFP URA3:p6LacO-mrsmtINO1 }\end{array}$ & Figure 3B \\
\hline DBY362 & $\begin{array}{l}\text { MATa/MAT a ade2-1/ade2-1 can1-100/can1-100 his3-11,15/ HIS3:Lacl-GFP leu2-3,112/ } \\
\text { LEU2:TetR-GFP trp1-1/trp1-1 ura3-1/ URA3:p6Lac0128-MRS50 INO1/INO1:TetO-Nat } \\
\text { SEC63/SEC63-13myc::Kan }\end{array}$ & Figure 3C \\
\hline DBY515 & 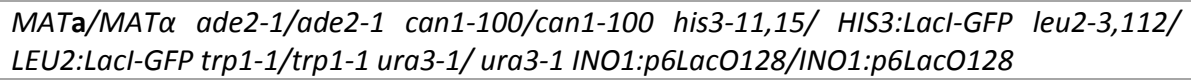 & Figure $5 A$ and $B$ \\
\hline DBY766 & 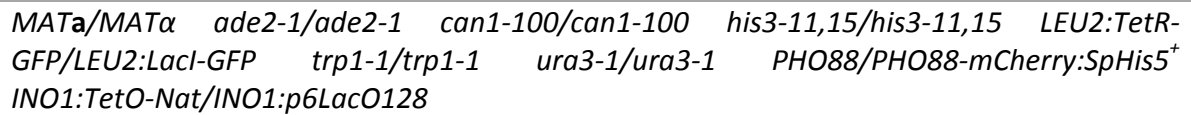 & Figure 6 \\
\hline DBY768 & 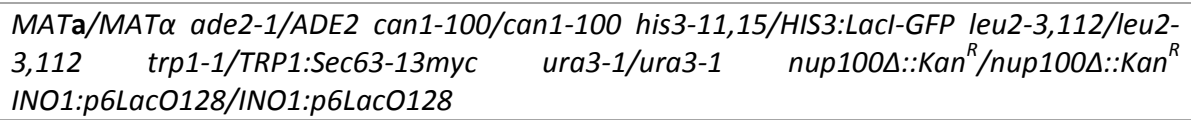 & Figure $5 C$ and $D$ \\
\hline DBY796 & $\begin{array}{l}\text { MATa ade2-1 can1-100 his3-11,15 leu2-3,112 trp1-1 ura3-1 INO1:TetO-Nat LEU2:TetR-GFP } \\
\text { HIS3:Lacl-GFP URA3:GRS II-p6LacO128 }\end{array}$ & Figure 4D \\
\hline DBY798 & $\begin{array}{l}\text { MATa ade2-1 can1-100 his3-11,15 leu2-3,112 trp1-1 ura3-1 INO1:TetO-Nat LEU2:TetR-GFP } \\
\text { HIS3:Lacl-GFP URA3:p6Lac0128-INO1 put3A }\end{array}$ & Figure 4B \\
\hline
\end{tabular}


Please cite this article as: Donna Garvey Brickner, Robert Coukos and Jason H. Brickner (2015). INO1 transcriptional memory leads to DNA zip code-dependent interchromosomal clustering. Microbial Cell 2(12): 481-490. doi: 10.15698/mic2015.12.242

\section{REFERENCES}

1. Meldi L \& Brickner JH (2011). Compartmentalization of the nucleus. Trends Cell Biol 21: 701-708.

2. Brickner JH and Walter $P$ (2004). Gene recruitment of the activated INO1 locus to the nuclear membrane. PLoS Biol. 2:e342.

3. Casolari JM, Brown CR, Komili S, West J, Hieronymus $\mathrm{H}$ and Silver PA (2004). Genome-wide localization of the nuclear transport machinery couples transcriptional status and nuclear organization. Cell 117, 427439.

4. Dieppois $\mathrm{G}$, Iglesias $\mathrm{N}$ and Stutz $F$ (2006). Cotranscriptional recruitment to the mRNA export receptor Mex67p contributes to nuclear pore anchoring of activated genes. Mol Cell Biol. 26:7858-7870.

5. Taddei A, Van Houwe G, Hediger F, Kalck V, Cubizolles F, Schober H and Gasser SM (2006). Nuclear pore association confers optimal expression levels for an inducible yeast gene. Nature 441:774-778.

6. Ahmed S, Brickner DG, Light WH, Cajigas I, McDonough M, Froyshteter AB, Volpe T and Brickner JH (2010). DNA zip codes control an ancient mechanism for gene targeting to the nuclear periphery. Nat Cell Biol 12:111-118.

7. Brickner DG, Ahmed S, Meldi L, Thompson A, Light WH, Young M, Hickman TL, Chu F, Fabre E and Brickner JH (2012). Transcription factor binding to a DNA zip code controls interchromosomal clustering at the nuclear periphery. Dev Cell 22:1234-1246.

8. Brickner DG, Cajigas I, Fondufe-Mittendorf Y, Ahmed S, Lee PC, Widom J and Brickner JH (2007). H2A.Z-mediated localization of genes at the nuclear periphery confers epigenetic memory of previous transcriptional state. PLoS Biol 5:e81.

9. Cabal GG, Genovesio A, Rodriguez-Navarro S, Zimmer C, Gadal O, Lesne $A$, et al. (2006). SAGA interacting factors confine sub-diffusion of transcribed genes to the nuclear envelope. Nature 441:770-773.

10. Light WH, Brickner DG, Brand VR and Brickner JH (2010). Interaction of a DNA zip code with the nuclear pore complex promotes H2A.Z incorporation and INO1 transcriptional memory. Mol Cell 40:112-125.

11. Casolari JM, Brown CR, Drubin DA, Rando OJ. and Silver PA (2005). Developmentally induced changes in transcriptional program alter spatial organization across chromosomes. Genes Dev 19:1188-1198.

12. Brown CR, Kennedy CJ, Delmar VA, Forbes DJ and Silver PA (2008). Global histone acetylation induces functional genomic reorganization at mammalian nuclear pore complexes. Genes Dev 22:627-639.

13. Capelson M, Liang Y, Schulte R, Mair W, Wagner U and Hetzer MW (2010). Chromatin-bound nuclear pore components regulate gene expression in higher eukaryotes. Cell 140:372-383.

14. Kalverda B, Pickersgill H, Shloma VV and Fornerod M (2010). Nucleoporins directly stimulate expression of developmental and cellcycle genes inside the nucleoplasm. Cell 140:360-371.

15. Light WH, Freaney J, Sood V, Thompson A, D'Urso A, Horvath C and Brickner JH (2013). A conserved role for human Nup98 in altering chromatin structure and promoting epigenetic transcriptional memory. PLoS Biology 11:e1001524.
16. Brickner DG and Brickner JH (2010). Cdk phosphorylation of a nucleoporin controls gene localization through the cell cycle. Mol Biol Cell 21:3421-3432.

17. Brickner DG and Brickner JH (2011). Gene positioning is regulated by phosphorylation of the nuclear pore complex by Cdk1. Cell Cycle 10: 392-395.

18. Gotta M, Laroche T, Formenton A, Maillet L, Scherthan H and Gasser SM (1996). The clustering of telomeres and colocalization with Rap1, Sir3, and Sir4 proteins in wild-type Saccharomyces cerevisiae. J Cell Biol 134, 1349-1363.

19. Thompson M, Haeusler RA, Good, PD and Engelke DR (2003). Nucleolar clustering of tRNA genes. Science 302:1399-1401.

20. Lanzuolo C, Roure V, Dekker J, Bantignies F and Orlando V (2007) Polycomb response elements mediate the formation of chromosome higher order structures in the bithorax complex. Nat Cell Biol 10:11671174

21. Tolhuis B, Blom M, Kerkhoven RM, Pagie L, Tenissen $H$, Nieuwland $M$, Simonis $M$, de Laat $W$, van Lohuizen $M$ and van Steensel B (2011). Interactions among Polycomb domains are guided by chromosome architecture. PLoS Genet 3:e1001343.

22. Brown JM, Leach J, Reittie JE, Atzberger A, Lee-Prudhoe J, Wood WG, Higgs DR, Iborra FJ and Buckle VJ (2006). Coregulated human globin genes are frequently in spatial proximity when active. J Cell Biol $172,177-187$

23. Schoenfelder S, Sexton T, Chakalova L, Cope NF, Horton A, Andrews S, Kurukuti S, Mitchell JA, Umlauf D, Dimitrova DS, Eskiw $\mathrm{CH}$, Luo Y, Wei CL, Ruan Y, Beiker JJ and Fraser P (2010). Preferential associations between co-regulated genes reveal a transcriptional interactome in erythroid cells. Nat Genet 42, 53-61.

24. $X u$ M and Cook PR (2008). Similar active genes cluster in specialized transcription factories. J Cell Biol 181, 615-623.

25. Longtine MS, McKenzie A, 3rd, Demarini DJ, Shah NG, Wach A, Brachat A, Phillipsen $P$ and Pringle $J$ (1998). Additional modules for versatile and economical PCR-based gene deletion and modification in Saccharomyces cerevisiae. Yeast 14: 953-961.

26. Amberg DC, Burke DJ and Strathern J (2005). Methods in Yeast Genetics: A Cold Spring Harbor Laboratory Course Manual. Cold Spring Harbor Press, Cold Spring Harbor, NY, USA.

27. Brickner DG, Light W and Brickner JH (2010). Quantitative localization of chromosomal loci by immunofluorescence. Meth Enzymol 470: 571-582.

28. Egecioglu DE, D'Urso A, Brickner DG, Light WH and Brickner JH (2014) Approaches to studying subnuclear organization and genenuclear pore interactions. Methods Cell Biol 122:463-485. 